\title{
GPS Carrier-Phase Time Transfer Using Single-Difference Integer Ambiguity Resolution
}

\author{
J. Delporte, F. Mercier, D. Laurichesse, and O. Galy \\ CNES_French Space Agency, 18 avenue Edouard Belin, Toulouse Cedex 4 31401, France
}

Correspondence should be addressed to F. Mercier, flavien.mercier@cnes.fr

Received 13 July 2007; Revised 25 October 2007; Accepted 15 January 2008

Recommended by Demetrios Matsakis

GPS is widely used for time and frequency transfer. To estimate the clock difference between two ground stations, a single baseline solution can be performed using external products for the modelling of the geometrical effects (constellation ephemeris and station coordinates). The baseline solution relies on a single-difference formulation, using code and phase ionosphere-free measurements. The phase ambiguities are usually adjusted as floating parameters. Such solutions give very good results when modelling hypotheses are consistent between the external solution (e.g., GPS orbits) and the baseline solution. However, the frequency bias in the computed clock is very sensitive to discrepancies in the models, and is only observed thanks to the code measurement, with limitations due to the noise. Here, we propose to solve the integer ambiguities on single-difference phase measurements. The advantage is the complete elimination of the clock drifts observed in floating ambiguities solutions. This formulation allows also a reliable continuous connection between overlapping clock solutions (jumps between such solutions can be completely eliminated). Several time transfer results are analyzed and compared to TWSTFT. The methodology has been extended to a network of stations using integer ambiguities on zero-difference measurements. The corresponding results are given for a few European stations.

Copyright ( 92008 J. Delporte et al. This is an open access article distributed under the Creative Commons Attribution License, which permits unrestricted use, distribution, and reproduction in any medium, provided the original work is properly cited.

\section{INTRODUCTION}

GPS time transfer is known to be an accurate way to compare ground clocks. An extensive analysis of the various techniques is given in [1]. The discontinuities at day boundaries observed in the current IGS precise time and geodesy solutions degrade the precision of their time and frequency solutions, but also provide valuable information about their timing accuracy [2]. In [1], two methods are proposed to deal with this problem: clock handover and ambiguity stacking. The drawback of these methods is that many internal parameters must be kept with each individual daily solution to compute a global continuous clock solution (e.g., normal equations and ambiguities of the overlapping passes). These methods are, therefore, not usable by external users who only have access to the daily ephemeris and clocks solutions. Independent time and frequency transfer applications need a method not so strongly related to the internal characteristics of a given global solution.

Here, we will first focus on single baseline solutions (time and frequency transfer between two stations). The GPS code measurement provides information on the clock difference between the two stations. The performance is limited for short durations by the noise of the code. The results can be improved by adding phase measurements that are much less noisy but whose use has some drawbacks. This is interesting for short durations (typically below ten days).

The single baseline solution is performed using external products for the modeling of the geometrical effects (constellation ephemeris and stations coordinates, e.g., IGS products [3]). These precise products rely generally on double difference processing with integer ambiguity fixing.

The single difference ionosphere-free code and phase measurements are processed to obtain the clock difference at each epoch. The clock difference between the two receivers is solved together with one floating-phase ambiguity for each pass [4]. The frequency bias of the solution is very sensitive to the model precision. Such biases appear even in very good global solutions, which use double-difference ambiguity fixing (see [2, Figure 8]). These frequency biases are due to small errors accumulated on the floating zero-difference ambiguities. It is shown here that when the integer nature of the ambiguity is taken into account on single- or zero-difference measurements, these frequency biases are totally suppressed. 
Such properties were shown in [5] on short baselines and single-difference measurements. Simulations on long baselines [6] have also shown that taking into account integer ambiguities minimizes the magnitude of frequency biases in the solutions.

Integer ambiguity fixing is a well-developed method in precise geodetic applications [1]. In these applications, the integer ambiguity fixing is performed on double-difference measurements. This process has the advantage of eliminating clocks from the solution leading to smaller problems to solve. Other propagation common errors are also reduced in the process depending on the relative geometry. When an integer constraint on double-difference ambiguities is added to the global clock solution, the solution is stabilized making it more robust to potential mismodeling $[5,7]$.

Different methods have already been developed for integer ambiguity searching like the LAMBDA method [8], but the latter is more focused on real-time data processing for precise positioning. However, in these geodetic applications, the integer ambiguity property is only considered on doubledifference data.

The approach presented here uses a new formulation allowing integer ambiguity fixing on single-difference measurements while solving for clock offsets at each epoch. It is well adapted for post-processed least-square analysis on a single baseline.

The method has been extended to more than two stations, giving global clock solutions with integer ambiguity fixing on a station network. In this case, the ambiguities are defined at zero-difference level $[9,10]$. Several time-transfer results using the single baseline [11] and the network methods are presented in this paper and compared to TWSTFT and to IGS clock products.

\section{AMBIGUITY RESOLUTION METHOD}

\subsection{Methodology}

For a given baseline, the general methodology to estimate the time offset between the two ground stations is as follows:

(i) absolute positioning for both stations using IGS precise ephemeris and clocks (precise point positioning (PPP) or "all-in-view" technique).

(ii) relative solution ("common-view" technique) with estimation of the integer ambiguities and clock difference.

The absolute positioning solution is a floating-ambiguity solution using ionosphere-free code and phase combinations. The troposphere propagation delays, the station coordinates (if necessary), and the receiver clock offset relative to the time of the IGS constellation solution are computed. The purpose of this solution is to obtain a precise model for the propagation distance of the ionosphere-free signal.

The relative solution uses a single-difference formulation. Integer ambiguities (one value per pass) and clock differences are solved simultaneously. The station coordinates and the troposphere delays are held fixed to the values resulting from the absolute positioning step.

\subsection{Model equations}

Initial preprocessing is carried out by sequential finite differences on the measurements. Passes are defined as continuous phase measurements on the two frequencies. Phase measurements are then roughly aligned on code measurements by adding an integer number of cycles constant per pass. Pseudorange measurements are noted $p_{1}$ and $p_{2}$ for the two frequencies $f_{1}$ and $f_{2}$, respectively; $L_{1}$ and $L_{2}$ denote the corresponding aligned phase measurements.

By combining these data, the ionosphere-free combinations for code $\left(p_{c}\right)$ and phase $\left(Q_{c}\right)$ can be constructed:

$$
P_{c}=\frac{P_{2}-\gamma P_{1}}{1-\gamma}, \quad Q_{c}=\frac{\lambda_{2} L_{2}-\gamma \lambda_{1} L_{1}}{1-\gamma},
$$

where $\lambda_{1}$ and $\lambda_{2}$ are the wavelengths corresponding to $f_{1}$ and $f_{2}$, and $\gamma$ is the ratio $f_{1}^{2} / f_{2}^{2}$.

To model measurements and to compute partial derivatives with respect to station coordinates and vertical troposphere delays, it is necessary to know the geometry of the problem. IGS ephemeris and clocks are used to compute this geometry. The model equations for the ionosphere-free data are

$$
\begin{gathered}
P_{c}=D_{\text {geo }}+D_{\text {tropo }}+\left(h_{\text {rec }}-h^{\mathrm{emi}}\right)+\mu_{\text {rec }}^{\mathrm{emi}} \\
Q_{c}=D_{\text {geo }}+D_{\text {tropo }}+D_{\text {wind }}+\left(h_{\text {rec }}-h^{\mathrm{emi}}\right)-\frac{\lambda_{2} N_{2}-\gamma \lambda_{1} N_{1}}{1-\gamma},
\end{gathered}
$$

where $D_{\text {geo }}$ is the geometrical distance between the ionosphere-free phase centers of the transmitter and the receiver antennas, $D_{\text {wind }}$ corresponds to the effect of the relativephase rotation between the antennas of the transmitter and receiver (wind-up effect), and $D_{\text {tropo }}$ is the troposphere propagation delay. $N_{1}$ and $N_{2}$ are the unknown integer ambiguities on each frequency, constant over a pass. $h_{\text {rec }}$ is the receiver clock offset and $h^{\text {emi }}$ the transmitter clock offset.

$\mu_{\mathrm{rec}}^{\mathrm{emi}}$ is an unknown bias which reflects the fact that code and phase may be misaligned [9]. We use phase as the reference for our clocks, so $\mu_{\mathrm{rec}}^{\mathrm{emi}}$ represents the code bias. This contributes to the code-phase bias identified in [1].

\subsection{Absolute positioning}

This step consists in estimating the station coordinates and the zenith troposphere delays while identifying a floating ambiguity per pass. The receiver clock offset with respect to the reference time (here IGS time) at each epoch is also adjusted. No constraint is applied on the coordinates or on the clock. Zenith troposphere delays are adjusted every two hours assuming a linear evolution in-between. The delays are constrained according to $\left(T r_{i+1}-T r_{i}\right)^{2} / \sigma^{2}$, where $T r_{i}$ is the $i$ th zenith troposphere delay and $\sigma$ is equal to $5 \mathrm{~mm}$. 


\subsection{Relative solution}

It is shown in $[9,12]$ that the difference between the ambiguities $N_{2}$ and $N_{1}$ (widelane ambiguity) can be represented as

$$
N_{w}=N_{2}-N_{1}=f\left(P_{1}, P_{2}, L_{1}, L_{2}\right)+\left(a_{i}-a^{j}\right),
$$

where $a_{i}$ and $a^{j}$ are biases related, respectively, to the receiver $i$ and transmitter $j . f\left(P_{1}, P_{2}, L_{1}, L_{2}\right)$ is the four observables Melbourne-Wübenna linear combination. When computing the station-station single difference, the satellite contribution $a^{j}$ vanishes and the equation becomes ( $\Delta$ is the singledifference operator)

$$
\Delta N_{w}=\Delta N_{2}-\Delta N_{1}=f\left(\Delta P_{1}, \Delta P_{2}, \Delta L_{1}, \Delta L_{2}\right)+a_{i_{1} i_{2}},
$$

where $a_{i_{1} i_{2}}$ is the difference between receivers $i_{1}$ and $i_{2}$ biases. For most geodetic receivers, $a_{i}$ biases are slowly varying and their excursion remains small (typically below 0.1 widelane cycle) and thus can be estimated over the whole dataset [9] up to an arbitrary integer constant. It is then possible to compute the $\Delta N_{W}$ values for each pass using (4).

The model equations for the single-difference problem are derived from (2). Using (4), $\Delta N_{2}$ can be expressed as the sum of $\Delta N_{W}$ and $\Delta N_{1}$ leading to

$$
\begin{gathered}
\Delta P_{c}=\Delta\left(D_{\text {geo }}+D_{\text {tropo }}\right)+\Delta h_{\text {rec }}, \\
\Delta Q_{c}+\frac{\lambda_{2}}{1-\gamma} \Delta N_{w}=\Delta\left(D_{\text {geo }}+D_{\text {tropo }}+D_{\text {wind }}\right)+\Delta h_{\text {rec }}-\lambda_{c} \Delta N_{1}, \\
\lambda_{c}=\frac{\lambda_{2}-\gamma \lambda_{1}}{1-\gamma},
\end{gathered}
$$

where $\Delta h_{\text {rec }}$ is the difference between the receiver clocks and $\lambda_{c}$ is the ionosphere-free wavelength $(10.7 \mathrm{~cm})$. The outputs of the absolute positioning step are used to compute the geometrical quantities on the right-hand side. The troposphere is held fixed at the values previously adjusted (this provides a better observability, since all satellites in view are used.) Given the size of the wavelength and the precision of the geometrical models (a few centimeters), it is then possible to solve for integer values of $\Delta N_{1}$.

The computation of the integer ambiguities is done sequentially by aligning each pass with the preceding ones (using only the ionosphere-free phase equation). The phase clock difference $\Delta h_{\text {phase }}$ is then obtained using the computed integer $\Delta N_{1}$ values,

$$
\begin{aligned}
\Delta h_{\text {phase }}=\langle\Delta & Q_{c}+\frac{\lambda_{2}}{1-\gamma} \Delta N_{w}+\lambda_{c} \Delta N_{1} \\
& \left.-\Delta\left(D_{\text {geo }}+D_{\text {tropo }}+D_{\text {wind }}\right)\right\rangle_{\text {epoch }}
\end{aligned}
$$

(mean of the contribution of all satellites at a given epoch).

Because of the uncertainty in the integer ambiguity for the first pass, the $\Delta h_{\text {phase }}$ clock differences are also defined up to an integer number of $\lambda_{c}$. Clock solutions computed in different batches may differ by an integer number of $\lambda_{c}$, however, when datasets overlap, it is possible to realign the solutions. This means that continuous long-duration frequency

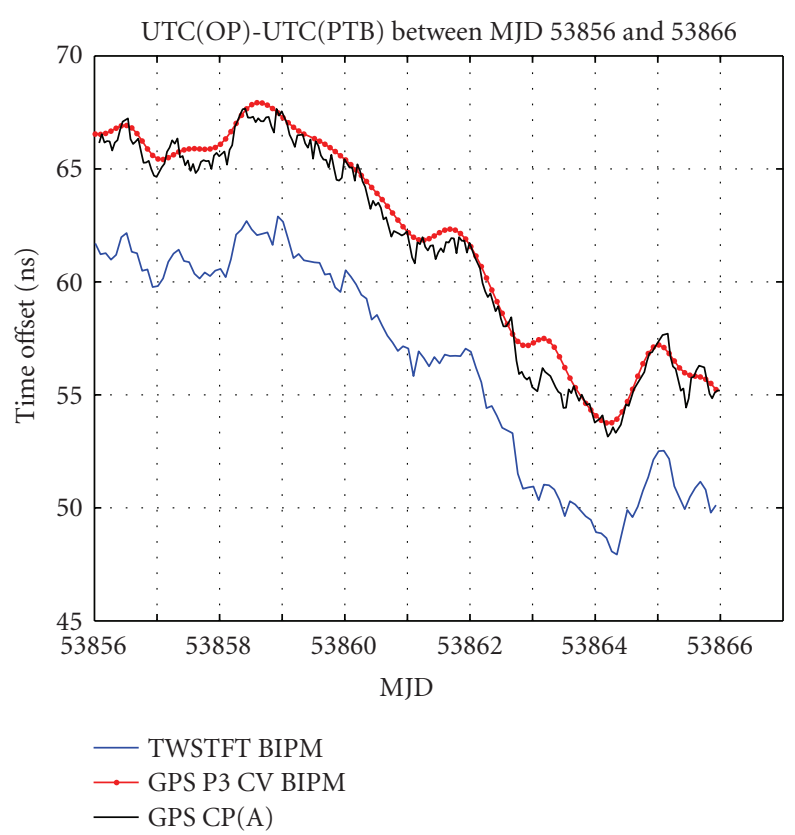

FIgURE 1: Time offset UTC(OP)-UTC(PTB) computed by three different techniques using the single baseline approach.

transfer solutions can be constructed simply by solving the integer $\lambda_{c}$ jumps at overlaps.

Because $a_{i}$ biases are only known up to an arbitrary integer constant, a bias is left in $\Delta h_{\text {phase }}$. This bias can be adjusted using code measurements in order to achieve time transfer (and not only frequency transfer). We will refer to this clock difference as GPS carrier phase with integer ambiguity, $\mathrm{CP}(\mathrm{A})$. The advantage of our approach is that this final bias can be adjusted over the length of the complete data set (if there are no phase interruptions), leading to a significant reduction in the uncertainties.

\section{TIME TRANSFER RESULTS}

\subsection{Continental baseline (OPMT/PTBB, $700 \mathrm{~km})$}

OPMT is an IGS station located in Paris Observatory; it is driven by a Hydrogen Maser which is compared to UTC(OP) on an hourly basis (these corrections were provided by Paris Observatory). The receiver is an Ashtech Z-12T. The station is calibrated so that we can relate the measurements to $\mathrm{UTC}(\mathrm{OP})$.

PTBB is also an IGS station located in PTB (Braunschweig, Germany); it is directly driven by UTC(PTB). Calibration delays for both stations have been used for the computation of GPS CP(A). Therefore, a GPS time transfer between these stations allows to compute UTC(OP)UTC(PTB). This offset is also computed by BIPM using TWSTFT and GPS P3 CV (common-view) using Vondrak smoothing. The BIPM data have been retrieved from their ftp site [13].

In Figure 1, we compare the TWSTFT and GPS P3 CV from BIPM data to our GPS CP(A) time transfer. 


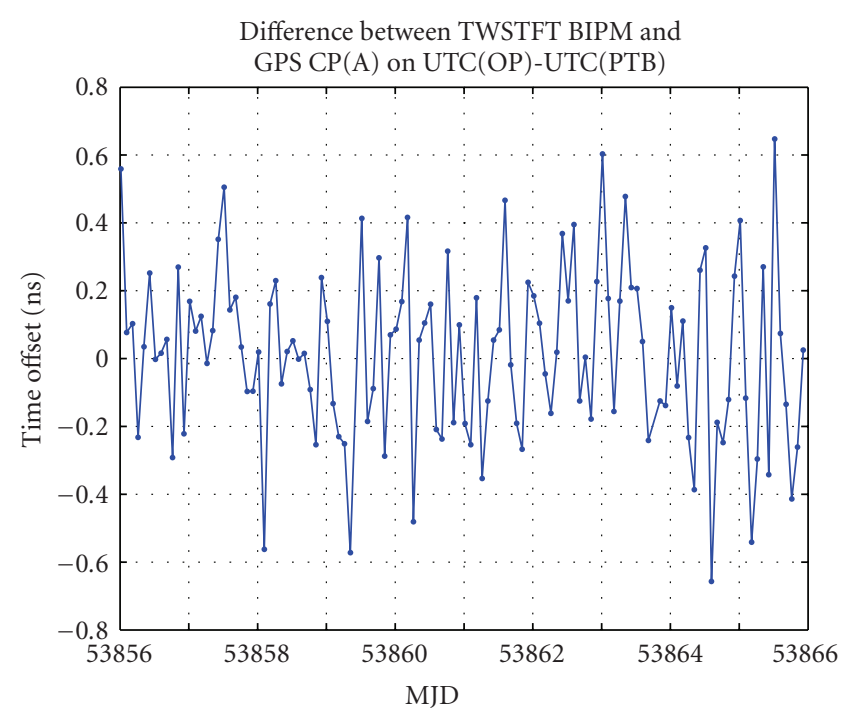

Figure 2: Difference between BIPM TWSTFT and GPS CP(A).

We observe a 5-nanosecond bias between the TWSTFT and the two GPS solutions. Short-term variations are very similar to TWSTFT and GPS CP(A), while they are invisible with GPS P3 CV with Vondrak smoothing.

In Figure 2, the difference between BIPM TWSTFT and GPS CP $(A)$ is presented in the same period.

The standard deviation of the difference between TWSTFT and GPS CP(A) is 0.25 nanosecond, with no drift. A constant bias of 5 nanoseconds has been removed from the data.

Figure 3 shows the stability of UTC(OP)-UTC(PTB) computed with TWSTFT, GPS P3 CV from BIPM data, and CNES GPS CP(A).

The frequency stabilities of the time offset UTC $(O P)$ UTC(PTB) computed by TWSTFT and GPS CP(A) agree very well. The apparent good short-term stability of the BIPM GPS P3 CV is only due to the smoothing and is not deemed to be representative of the clock difference behavior.

\subsection{Transatlantic baseline (USN3/PTBB, $\sim 6300 \mathrm{~km}$ )}

USN3 is an IGS station located at the US Naval Observatory; it is driven by UTC(USNO) with no corrections to apply (see USN3 IGS log file [14]).

The goal is to perform the GPS time transfer between the USN3 and PTBB stations to compute UTC(USNO)$\mathrm{UTC}(\mathrm{PTB})$ and compare results to the BIPM data. For such a long baseline, a direct ambiguity solution is not possible because modeling errors are large (in particular troposphere modeling at low elevation). In addition single difference passes are shorter, and there are few simultaneous passes. In order to apply our ambiguity resolution method, we introduce two bridge stations (STJO and REYK). This leads to three short baselines $(\sim 2500 \mathrm{~km})$.

The three baselines are processed independently (but with the same geometrical parameters), giving estimates for

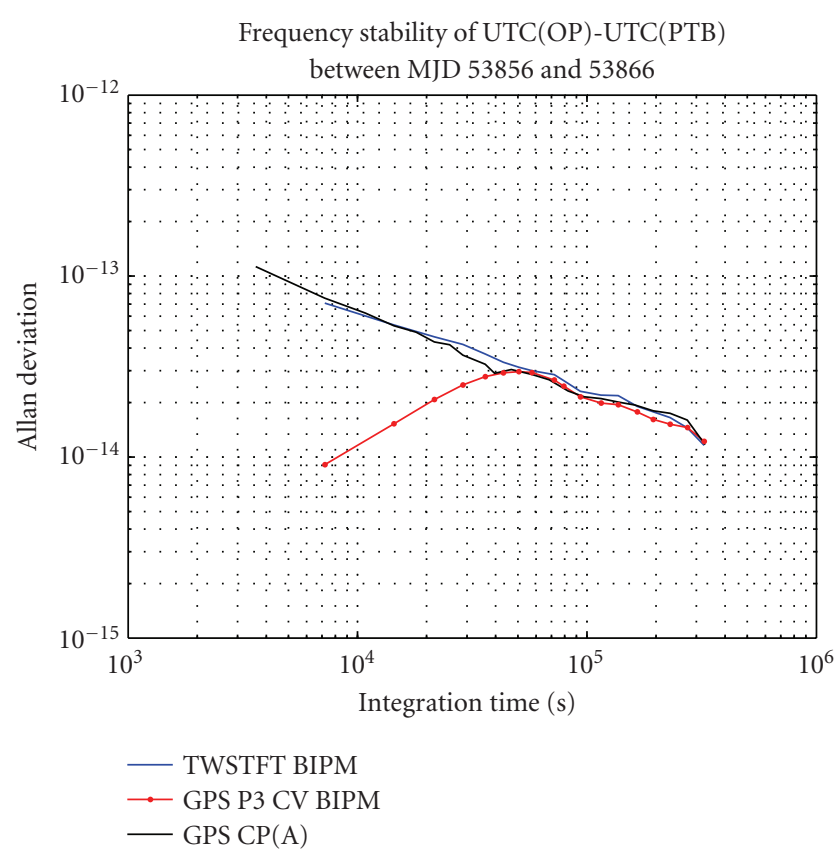

FIgUre 3: Allan deviation of the time offset UTC(OP)-UTC(PTB) for the three different techniques.

TABLE 1: N1 ambiguities on the three baselines.

\begin{tabular}{lccc}
\hline Baseline & Length $(\mathrm{km})$ & Total N1 & Integer N1 $(\%)$ \\
\hline PTBB-REYK & 2265 & 231 & $191(83 \%)$ \\
REYK-STJO & 2597 & 230 & $199(87 \%)$ \\
STJO-USN3 & 2179 & 152 & $145(95 \%)$ \\
\hline
\end{tabular}

the three clock differences (6 days solution). Table 1 summarizes the results.

During the identification of the integer ambiguities, some passes have been rejected due to inconsistent overlaps (threshold used 0.25 cycle on the overlap between two passes).

The clock difference for the baseline PTBB/USN3 is obtained by adding the three contributions. There are eleven days of data that have been split into two batches of 6 days with one-day overlap. These results (with and without integer fixing) are shown in Figure 4.

As observed in [4, 5], floating solutions sometimes exhibit important drifts, which are not present in the integer resolution. In addition, the integer clocks differ exactly by one cycle on the central overlapping day. A continuous clock covering the whole period is then easily obtained by shifting the second solution by one cycle to align it with the first one. The remaining alignment error is then below one cycle (about 0.3 nanosecond).

In Figure 5, we compare the two BIPM techniques to the GPS CP(A) time transfer.

The same conclusions as for Figure 1 can be drawn. The standard deviation of the difference between the TWSTFT and GPS CP (A) on this baseline is 0.3 nanosecond. 


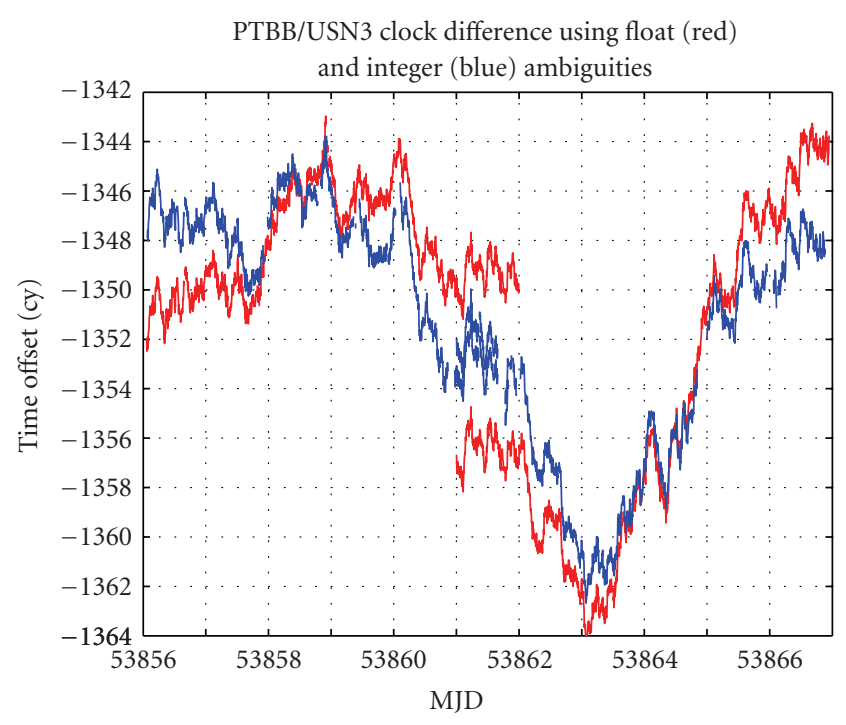

FIgURE 4: PTBB/USN3 clock difference for the two 6-day batches for integer (blue) and floating (red) solutions (units in cycles).
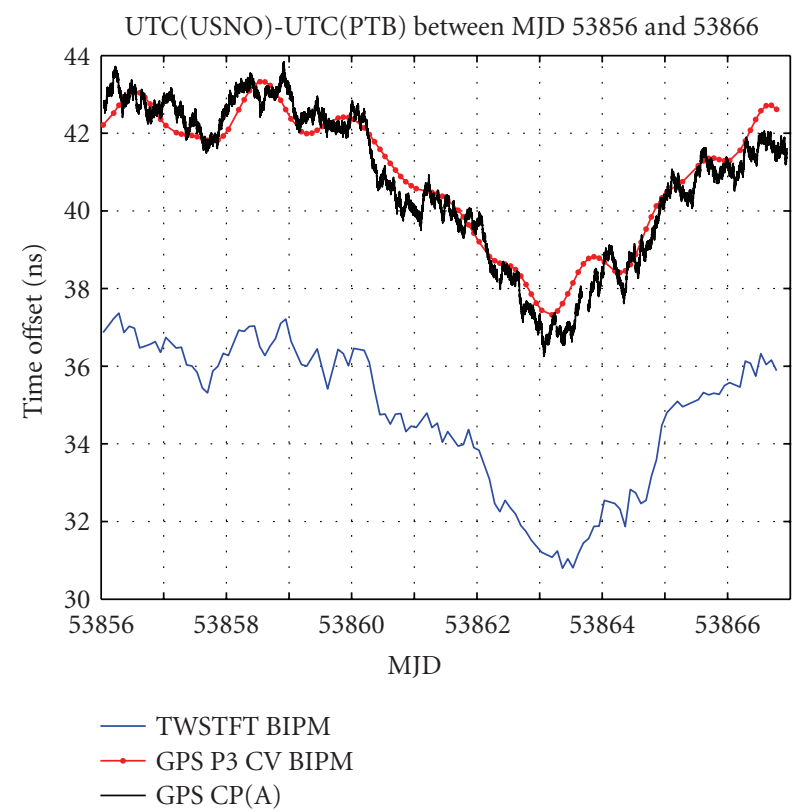

FIgURE 5: Time offset UTC(USNO)-UTC(PTB) computed by three different techniques using the single baseline approach.

\subsection{Network solution}

The method has been generalized to a network solution, which is detailed in $[9,10]$. The global solution approach is similar to the single baseline formulation extended to zerodifference measurements (identification of ground stations and GPS satellite clocks). This means that all receiver clocks are computed relative to a reference clock chosen in the network, modulo an integer number of $\lambda_{c}$ wavelengths. The corresponding $\mathrm{CP}(\mathrm{A})$ clock differences are obtained after alignment to code measurements. Figure 6 shows a map of the

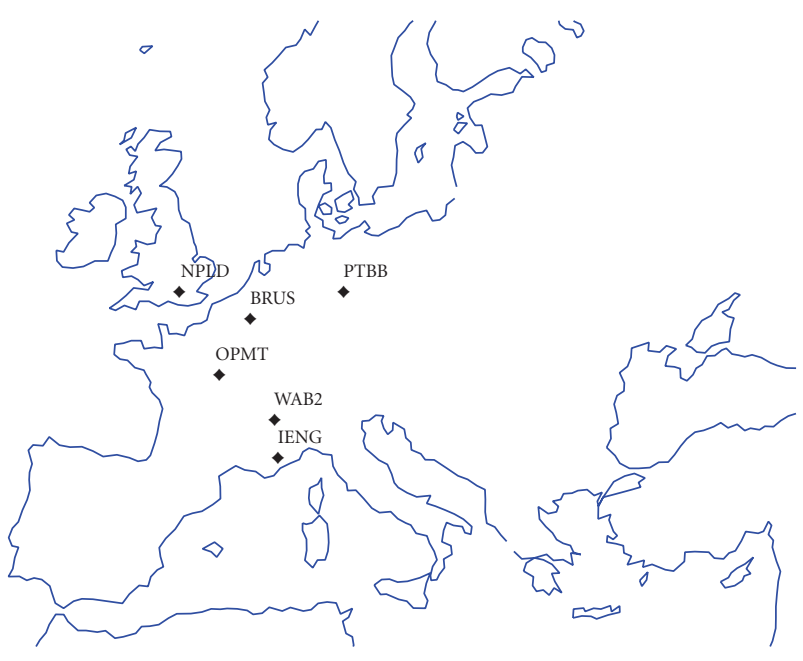

FiguRE 6: European network used in this study.

stations used in the solution. BRUS was chosen as time reference.

The station coordinates and zenith troposphere delays are forced to the values obtained from the absolute positioning that is performed independently for each station as for the single baseline solution.

Clocks and ambiguities are adjusted in the integer solution; $98 \%$ of $\mathrm{N} 1$ integer ambiguities are identified (statistics are computed only for passes with at least one common view with another station).

Figure 7 presents the time offset UTC $(\mathrm{OP})-\mathrm{UTC}(\mathrm{PTB})$ from BIPM TWSTFT data (blue circles), from IGS clock products (red dots), and from our network solution. We have subtracted the same arbitrary value for the two GPS solutions, because the receiver internal calibration delays were not available.

We notice a good agreement between the three solutions. Differences between BIPM TWSTFT and CP(A), and between IGS and CP(A), are shown in Figure 8 (TWSTFT has been interpolated every hour).

The standard deviation of the difference between GPS $\mathrm{CP}(\mathrm{A})$ and BIPM TWSTFT is 0.23 nanosecond. The standard deviation between the two GPS solutions is lower, however, we observe bias variations up to 1 nanosecond in the IGS clocks at day boundaries. Thus, the $\mathrm{CP}(\mathrm{A})$ solution noise is close to the IGS noise, while offering the same long term coherency as TWSTFT. Some errors below one cycle (0.3 nanosecond) may remain between the two GPS solutions because the current processing does not take into account possible code/phase biases.

\section{CONCLUSION}

A simple method for the resolution of integer ambiguities on zero- or single-difference measurements and the computation of the associated clocks has been presented and validated. 


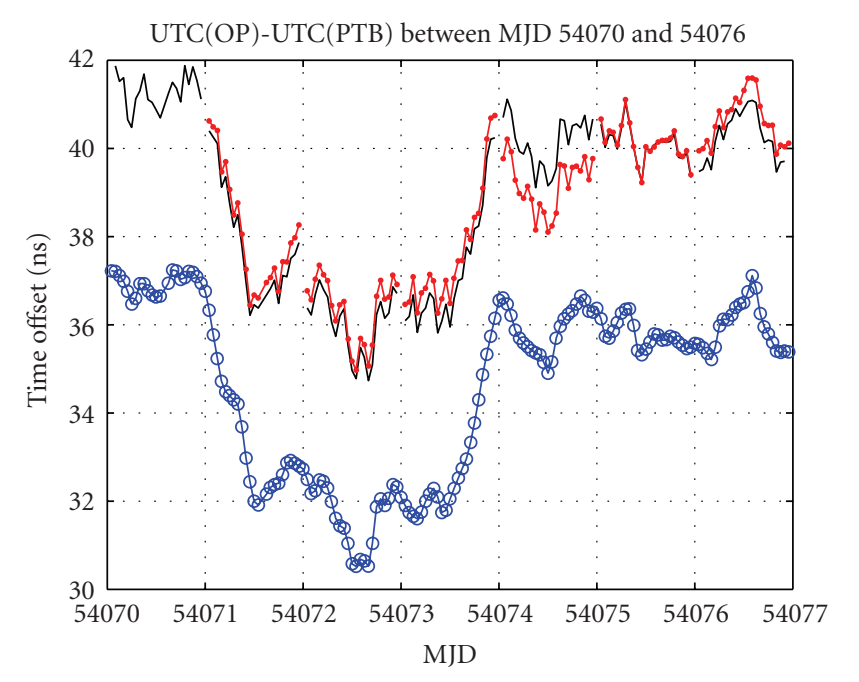

- GPS CP(A)
- IGS clock product
- TWSTFT BIPM

Figure 7: Time offset UTC(OP)-UTC(PTB) from BIPM TWSTFT, IGS clock products, and GPS CP(A).

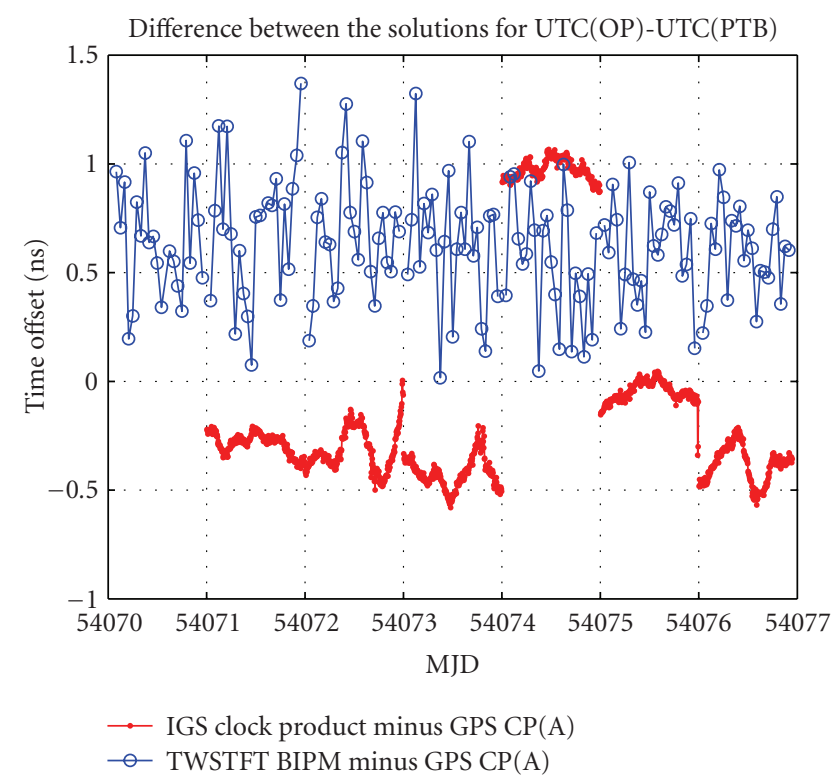

FIgURE 8: Difference between the solutions for UTC(OP)$\mathrm{UTC}(\mathrm{PTB})$ - referenced to GPS CP(A).

The main advantages are as follows.

(i) The phase clocks estimated with integer ambiguities are very stable over the long term: the drifts present in similar floating solutions disappear completely, and it is easy to handle day-boundary discontinuities.

(ii) The clock solution is self-sufficient; it uses only IGS precise ephemeris as external modeling input.

The results have been successfully compared to the BIPM TWSTFT on the OP-PTB and USNO-PTB baselines. Similar results are also shown in a European network of 6 stations.
Agreement with BIPM TWSTFT at the level of 0.3 nanosecond (standard deviation over 11 days) has been achieved even on transatlantic baselines. It would be of particular interest to repeat such a comparison over a longer time period in order to confirm this good agreement and to investigate possible seasonal effects.

The level of precision which has been obtained now allows the observation of receiver specific delays ("code/phase" biases). Further studies are necessary to characterize the code behavior and to determine new calibration procedures for the time receivers.

\section{ACKNOWLEDGMENTS}

The authors thank David Valat (Paris Observatory) for providing the files containing the offset between their Hydrogen Maser and UTC(OP). They are also grateful to Dr. J. P. Berthias for his helpful advice. The authors also thank the reviewers for their many constructive remarks.

\section{REFERENCES}

[1] R. Dach, T. Schildknecht, U. Hugentobler, L.-G. Bernier, and G. Dudle, "Continuous geodetic time-transfer analysis methods," IEEE Transactions on Ultrasonics, Ferroelectrics, and Frequency Control, vol. 53, no. 7, pp. 1250-1259, 2006.

[2] J. Ray and K. Senior, "IGS/BIPM pilot project: GPS carrier phase for time/frequency transfer and timescale formation," Metrologia, vol. 40, no. 4, p. 205, 2003.

[3] J. M. Dow, R. E. Neilan, and G. Gendt, "The international GPS service: celebrating the 10th anniversary and looking to the next decade," Advances in Space Research, vol. 36, no. 3, pp. 320-326, 2005.

[4] J. Delporte, F. Mercier, and Ph. Guillemot, "New frequency comparisons using GPS carrier phase at CNES," in Proceedings of 19th European Time and Frequency Forum (EFTF '05), pp. 568-573, Besancon, France, March 2005.

[5] C. Rieck, P. Jarlemak, and K. Jaldehag, "The use of ambiguity resolution for continuous real-time frequency transfer by filtering GNSS carrier phase observations," in Proceedings of the 20th European Frequency and Time Forum (EFTF '06), pp. 580-587, Braunschweig, Germany, March 2006.

[6] C. Hackman and J. Levine, "Towards sub-10-16 transcontinental GPS carrier-phase frequency transfer: a simulation study," in Proceedings of IEEE International Frequency Control Symposium and Exposition (IFCS '06), pp. 779-787, Miami, Fla, USA, July 2006.

[7] R. Dach, G. Beutler, U. Hugentobler, et al., "Time transfer using GPS carrier phase: error propagation and results," Journal of Geodesy, vol. 77, no. 1-2, pp. 1-14, 2003.

[8] P. Teunissen and C. Tiberius, "Integer least-squares estimation of the GPS phase ambiguities," in Proceedings of International Symposium on Kinematic Systems in Geodesy, Geomatics and Navigation, pp. 221-231, Banff, Canada, August-September 1994.

[9] F. Mercier and D. Laurichesse, "Receiver/Payload hardware biases stability requirements for undifferenced Widelane ambiguity blocking," Scientifics and fundamental aspects of the Galileo program Colloquium, Toulouse, France, October 2007. 
[10] D. Laurichesse and F. Mercier, "Integer ambiguity resolution on undifferenced GPS phase measurements and its application to PPP," ION GNSS-07, Fort Worth, Tex, USA, September 2007.

[11] J. Delporte, F. Mercier, D. Laurichesse, and O. Galy, "Fixing integer ambiguities for GPS carrier phase time transfer," in Proceedings of the IEEE International Frequency Control Symposium (IFCS '07), pp. 927-932, Geneva, Switzerland, May 2007.

[12] M. J. Gabor, "GPS carrier phase ambiguity, resolution using satellite-satellite single differences," in Proceedings of the Institute of Navigation (ION '99), Cambridge, Mass, USA, June 1999.

[13] ftp://tai.bipm.org/TimeLink/LkC/.

[14] http://igscb.jpl.nasa.gov/network/site/usn3.html. 

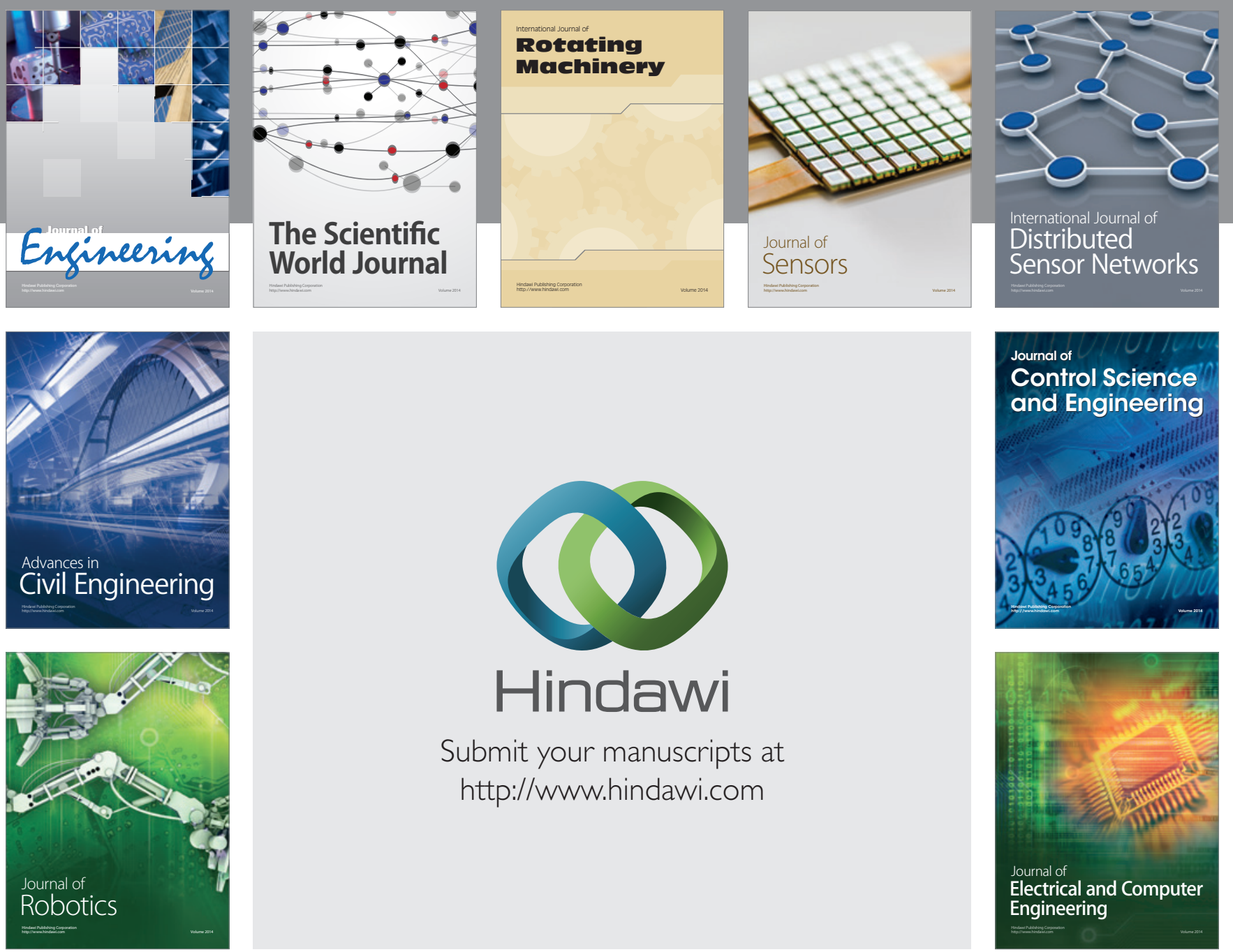

Submit your manuscripts at

http://www.hindawi.com
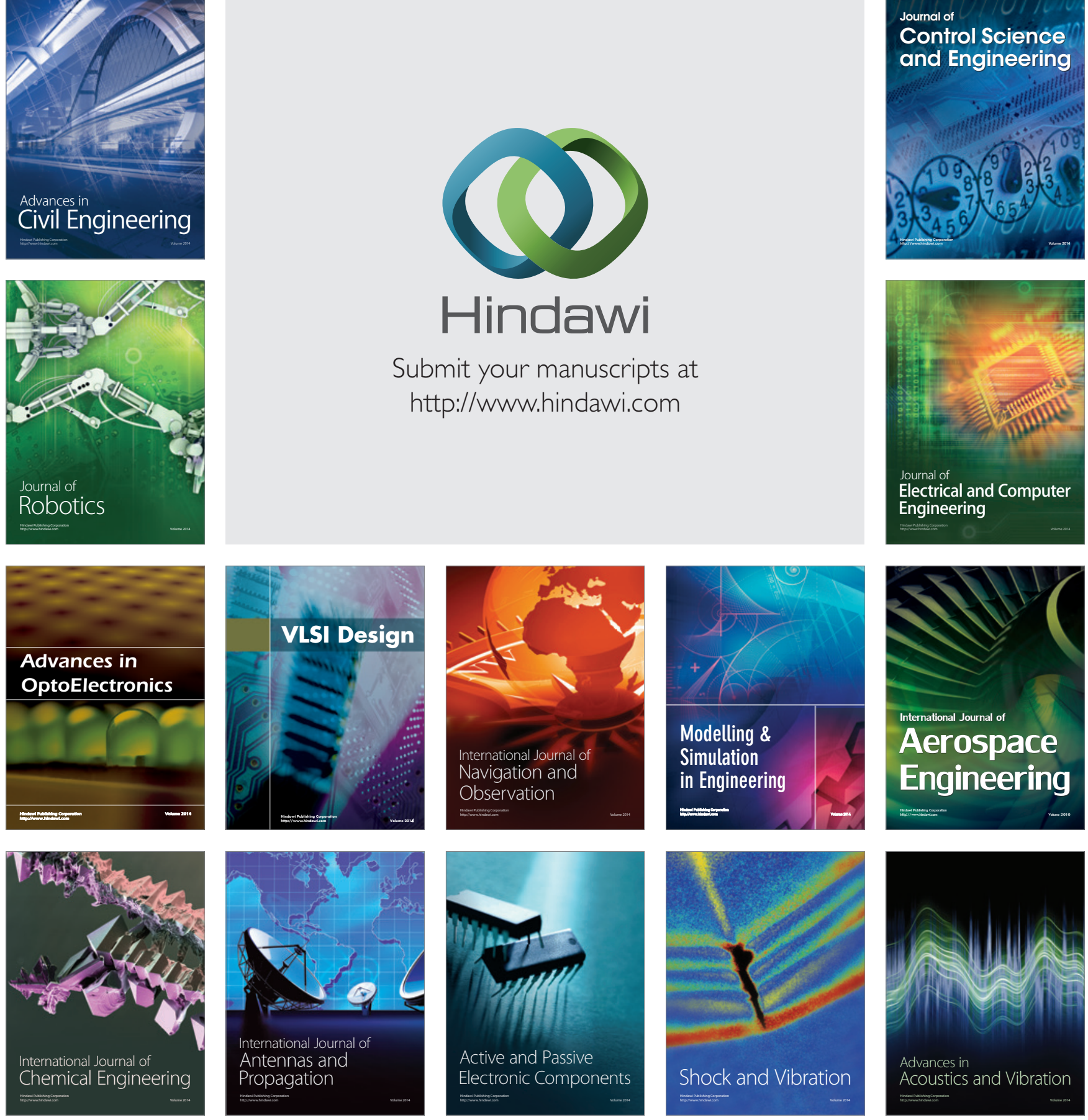